\title{
25 Research Soure \\ Quantitative Values from Synthetic MRI Correlate with Breast Cancer Subtypes
}

\section{Toshiki Kazama ( $\sim$ kazamat@fg7.so-net.ne.jp )}

Tokai University School of Medicine: Tokai Daigaku Igakubu Daigakuin Igaku Kenkyuka https://orcid.org/0000-0002-5588-4603

\section{Taro Takahara}

Tokai University School of Engineering Graduate School of Engineering: Tokai Daigaku Kogakubu Daigakuin Kogaku Kenkyuka

\section{Thomas C. Kwee}

University Medical Centre Groningen: Universitair Medisch Centrum Groningen

Noriko Nakamura

Tokai University School of Medicine: Tokai Daigaku Igakubu Daigakuin Igaku Kenkyuka Nobue Kumaki

Tokai University School of Medicine: Tokai Daigaku Igakubu Daigakuin Igaku Kenkyuka Naoki Niikura

Tokai University School of Medicine: Tokai Daigaku Igakubu Daigakuin Igaku Kenkyuka

\section{Tetsu Niwa}

Tokai University School of Medicine: Tokai Daigaku Igakubu Daigakuin Igaku Kenkyuka Jun Hashimoto

Tokai University School of Medicine: Tokai Daigaku Igakubu Daigakuin Igaku Kenkyuka

\section{Research Article}

Keywords: Magnetic resonance imaging, Breast neoplasms, Receptors, estrogen, Quantitative values

Posted Date: October 5th, 2021

DOI: https://doi.org/10.21203/rs.3.rs-879518/v1

License: (9) This work is licensed under a Creative Commons Attribution 4.0 International License. Read Full License 


\section{Abstract}

Purpose To correlate quantitative T1, T2, and proton density (PD) values from synthetic MRI with breast cancer subtypes.

Methods Twenty-eight breast cancer patients underwent MRI of the breast including synthetic MRI. T1, $\mathrm{T} 2$, and PD values were correlated with $\mathrm{Ki}-67 . \mathrm{T} 1, \mathrm{~T} 2$, and PD values were compared between estrogen receptor (ER) positive and ER negative cancers, and between Luminal A and Luminal B cancers. The effectiveness of $T 1, T 2$, and $P D$ in differentiating the ER-negative from the ER-positive group and Luminal A from Luminal B cancers was evaluated using receiver operating characteristic analysis.

Results Mean T2 relaxation of ER-negative cancers was significantly higher than that of ER-positive cancers $(p<.05)$. The T1, T2, and PD values exhibited a strong positive correlation with Ki-67 (Pearson's $r$ $=0.75,0.69$, and 0.60 respectively; $p<.001)$. Among ER-positive cancers $(n=23), T 1, T 2$, and PD values of Luminal A cancers were significantly lower than those of Luminal B cancers $(p<.05)$. The areas under the curve (AUCs) of T1, T2, and PD for discriminating ER-negative from ER-positive cancers were 0.74 (95\% confidence interval (Cl): 0.54-0.88), 0.87 (95\% Cl: 0.69-0.97), and 0.62 (95\% Cl: 0.42-0.79), respectively. The AUCs of T1, T2, and PD values for discriminating Luminal A from Luminal B cancers were 0.83 (95\% Cl: 0.61-0.95), 0.75 (95\% Cl: 0.52-0.90), and 0.75 (95\% Cl: 0.53-0.91), respectively.

Conclusion Quantitative values from synthetic MRI significantly correlate with subtypes of invasive breast cancers and may classify subtypes with reasonably good accuracy.

\section{Introduction}

Breast cancer is the most frequently diagnosed malignancy and the leading cause of cancer- related death among women [1]. Breast cancer is a heterogeneous disease with a high degree of diversity with regard to the risk of therapeutic resistance and disease progression [2]. Therefore, the need for individualized management is widely accepted $[2,3]$. In addition to the traditional parameters such as tumor size, grade, and lymph node status, the immunohistochemical-based classification is widely used $[2,3]$. Immunohistochemical assessments for tumor subtyping concern estrogen receptor (ER) and human epidermal growth factor receptor 2 (HER2) expressions, and proliferation according to the Ki-67 labeling index (Ki-67) $[2,3]$.

The presence of ER or progesterone receptor $(\mathrm{PgR})$ is a predictive marker of the long-term outcome and response to hormonal therapy [2,3]. Luminal-type (hormone receptor-positive) breast cancer is the most frequent subtype and is divided into two subtypes, namely Luminal A and Luminal $B$. Luminal $A$ is defined as a low-proliferation subtype $(\mathrm{Ki}-67<14)$, whereas Luminal $B$ is defined as a high-proliferation

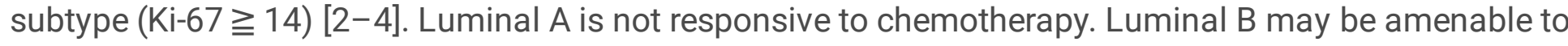
chemotherapy as well as endocrine therapy [2, 3]. For hormone receptor-negative breast cancer, chemotherapy frequently combined with molecular-targeted drugs may be useful [2,3]. Because these receptor expressions can change during treatment $[5,6]$, noninvasive assessment of receptor status, 
especially in recurrent lesions, may improve personalized treatment. As such, non-invasive assessment of Ki-67 may help select appropriate adjuvant therapy.

The recently developed technique of synthetic magnetic resonance imaging (MRI) allows for quantitative analysis of $\mathrm{T} 1$ relaxation, $\mathrm{T} 2$ relaxation, and proton density (PD) as well as generating various image contrasts using the information from one single scan [7-9]. Several investigators have reported that aggressive breast cancers tend to have high signal intensity on T2-weighted images [10-16]. We hypothesized that quantitative MR parameters such as T2 relaxation and proton density correlate to the tumor grade of breast cancers.

The purpose of this study was twofold: to correlate quantitative T1, T2, and PD values obtained using synthetic MR with tumor grade of breast cancers, and to determine if these quantitative values obtained using synthetic MR can differentiate subtypes of breast cancers.

\section{Materials And Methods}

\section{Subjects}

The prospective study protocol was approved by our institution's ethics committee. Between November 2020 and February 2021, patients with histologically proven invasive breast carcinoma, a tumor size larger than $15 \mathrm{~mm}$ on ultrasound, and who were referred for pretreatment evaluation, underwent breast MRI. Thirty consecutive patients were invited in this study and written informed consent was obtained from all participants.

\section{Mr Image Acquisition}

Studies were performed on a 1.5 T scanner (Ingenia, Philips Healthcare, Best, The Netherlands) using a 16-channel breast-phased array coil. Images were acquired in the prone position and in axial orientation to cover the whole breasts. Participating patients underwent synthetic MRI in addition to routine clinical MRI sequences. In synthetic MRI, quantification of T1 and T2 relaxation rates as well as PD was performed using the QRAPMASTER pulse method sequence, which is a multislice, multiecho, and multisaturation delay acquisition sequence [8]. Two sets of echo times (TEs) and 4 sets of delay times were used to generate 8 complex images in each section in order to quantify T1, T2, and PD. The TEs were 12.5 and $100 \mathrm{msec}$, the delay times were 151, 604, 2115, and $4382 \mathrm{msec}$, and the repetition time (TR) was $4462 \mathrm{msec}$. Thirty slices were acquired, voxel size was $1.42 \times 1.92 \times 4.00 \mathrm{~mm}^{3}$, and total acquisition time was 3:40 minutes. Synthetic images were created using SyMRI StandAlone software (SyntheticMR AB, Linköping, Sweden). Following synthetic MRI, routine T1-weighted images (TR/TE, 600/12 msec), STIR (TR/TE/TI, 3500/90/160 msec), diffusion-weighted images (DWI) using single-shot echo planar imaging (b values of 0 and $1500 \mathrm{~s} / \mathrm{mm}^{2}$; number of excitations, 1 and 14; TR/TE/TI, $6407 / 86 / 180 \mathrm{msec}$; field of view (FOV), $340 \mathrm{~mm}$; matrix, 112×112; section thickness, $4 \mathrm{~mm}$ ), and three- 
dimensional (3D) fat-suppressed dynamic contrast-enhanced MRI (TR/TE, 4.0/2.1 msec; flip angle, 15\%; FOV, $340 \mathrm{~mm}$; matrix, $352 \times 352$; slice thickness, $1 \mathrm{~mm}$; intersection gap, $0 \mathrm{~mm}$ ) were obtained. Dynamic scans were performed before and 4 times after the rapid injection of a bolus of $0.1 \mathrm{mmol} / \mathrm{L}$ of gadolinium-based contrast medium per kilogram of body weight.

\section{Image Analysis}

MR images were evaluated by a board-certified radiologist with 20 years of experience in breast MRI. Dedicated analysis software for synthetic MRI automatically calculated a value of R1 (i.e. 1/T1), R2 (i.e. $1 / \mathrm{T} 2$ ), and PD for all pixels in the ROI. The PD level of pure water was set at $100 \%$. Synthetic T1-weighted images (TR/TE, 600/10 msec) were made for measurement purposes. For measurement of T1, T2, and $\mathrm{PD}$, the radiologist selected the slice that showed the largest slice of the tumor on contrast-enhanced images. Then the corresponding slice was selected on synthetic T1-weighted images. A freehand region of interest (ROI) was placed on the synthetic T1-weighted image while cross-referencing to the contrastenhanced images, and with the radiologist being blinded to the clinical and pathologic information. A freehand ROI was placed just within the inner border of the tumor, in order to avoid the inclusion of surrounding fat. Mean R1, mean R2, and mean PD shown on the viewers were recorded. Mean T1 and mean T2 were calculated based on the following equation: $T 1=1 / R 1, T 2=1 / R 2$. Dedicated analysis software automatically calculated apparent diffusion coefficients (ADC) maps. The same radiologist measured the mean ADC of breast cancers in a similar manner with freehand ROls set on diffusionweighted images. The ROls were copied to the ADC maps to obtain the ADC value.

\section{Pathological Examination}

All pathological specimens were evaluated by our institution's pathology department using their standard clinical protocol. Pathologists evaluated the specimens on the basis of the World Health Organization histologic classification of breast tumors [17]. The expressions of ER and PgR were assessed by immunohistochemical staining. Per our institutional protocol, any expression of ER or PgR greater than $1 \%$ was considered positive $[18,19]$. Ki- 67 immunoreactivity was evaluated by using the percentage of immunoreactive tumor cells [20]. Based on $\mathrm{Ki}-67$, cancers were divided into low-proliferation group (Luminal A) $(\mathrm{Ki}-67<14)$ and the high-proliferation group (Luminal B) $(\mathrm{Ki}-67 \geqq 14)[3,4]$.

\section{Statistical Analysis}

Data were analyzed using MedCalc 19.8 (MedCalc, Ostend, Belgium). All data are expressed as means \pm standard deviation, unless otherwise specified. Normal distribution of the T1, T2, PD, and ADC values was tested using the Shapiro-Wilk test. Age, tumor diameter, Ki-67, and the quantitative MR parameters regarding ER status (positive vs. negative) and Luminal types (A vs. B) were compared using the unpaired $t$ test (for normally distributed data) or the Mann-Whitney U Test (for not normally distributed data). Quantitative MR parameters m including ADC, were correlated with Ki-67 using a Pearson's correlation 
analysis (for normally distributed data) or a Spearman correlation analysis (for not normally distributed data). Among resected tumors, the correlation of quantitative MR values with $\mathrm{Ki}-67$ of both biopsy specimen and surgical specimens was assessed.

The effectiveness of quantitative MR parameters, including ADC, in differentiating the ER-negative group from the ER-positive group and Luminal $B$ from the Luminal A was evaluated using receiver operating characteristic (ROC) analysis. For sensitivity and specificity, the optimal cut-off point was determined using Youden index. The area under the ROC curve was expressed as a mean and $95 \%$ confidence interval. All statistical tests were two-tailed and significance was set at a $\mathrm{P}<.05$.

\section{Results}

\section{Patients}

Thirty patients were invited, however, two of them refused to participate in this study. Finally, 28 female patients participated in the study (mean age $63.3 \pm 12.2$ years, range $32-80$ years). In one out of 28 patients, bilateral breast cancers were found. Therefore, a total of 29 breast cancers were analyzed. Eighteen patients underwent surgery and their surgical specimens were used for pathological analysis. The other 10 patients underwent preoperative chemotherapy and their biopsy specimens were used for pathological analysis, while tumor size was measured on contrast-enhanced MRI. The mean tumor size was $28 \mathrm{~mm}$ (range 12-70 mm). The histological classification of the tumors was as follows; 26 invasive carcinomas of no special type, 2 invasive lobular carcinomas, and 1 invasive micropapillary carcinoma. Most of the tumors were ER-positive (88\%) and all of the PgR-positive tumors also showed ER positivity. By combining immunohistochemical marker analysis, 10 (34\%) Luminal A tumors, 13 (45\%) Luminal B tumors, and $6(21 \%)$ ER-negative tumors were identified.

\section{Relationship With Er Status}

Clinical and pathological characteristics, and quantitative MR values for the 29 lesions are shown in Table 1. No statistical significance was found between the ER-positive cancers and the ER-negative cancers regarding age and tumor diameter. The Ki-67 of ER-negative cancers was significantly higher than that of ER-positive cancers $(p<.05)$. Mean T2 relaxation of ER-negative cancers $(92.1 \pm 13.0 \mathrm{msec})$ was significantly higher than that of ER-positive cancers ( $72.7 \pm 15.7 \mathrm{msec}, \mathrm{p}=.01)$ (Figs. 1-3). Mean T1 relaxation and PD of ER-negative cancers was higher than those of ER-positive cancers, but no significant difference was observed between them. The AUCs of T1, T2, and PD for the differentiation of ER-negative cancers from ER-positive cancers were 0.74 (95\% confidence interval (Cl): 0.54-0.88), 0.87 (95\% Cl: $0.69-0.97)$, and 0.62 (95\% Cl: 0.42-0.79), respectively (Fig. 4). The T2 value had the highest AUC, followed by T1 and PD. The optimal sensitivity and specificity of the T2 value were $100 \%$ and $78 \%$.

\section{Relationship With Ki-67}


The Shapiro-Wilk test showed that the T1, T2, PD, and ADC values followed a normal distribution. The $\mathrm{T} 1, \mathrm{~T} 2$, and PD derived from synthetic MR demonstrated a strong positive correlation with Ki-67 (Pearson's $r=0.75,0.69$, and 0.60 respectively; $p<.001$ ) (Fig. 5 and Table 2). Among 19 resected cancers, the correlation of biopsy specimen $\mathrm{Ki}-67$ and resected specimen $\mathrm{Ki}-67$ were demonstrated on Table 2. No significant difference was observed between them. No significant correlation was observed either between ADC and Ki-67 (Pearson's $r=0.11 ; p=.57$ ).

The clinical and pathological characteristics and quantitative MR values of ER-positive cancers $(n=23)$ are shown in Table 3. No statistical significance was found between the Luminal A and the Luminal $B$ cancers regarding tumor diameter. All T1 (991 $\pm 132 \mathrm{msec}$ vs. $1259 \pm 265 \mathrm{msec})$, T2 $(65.2 \pm 12.6 \mathrm{msec}$ vs. $78.5 \pm 15.8 \mathrm{msec})$, and PD values (63.4 $\pm 11.4 \%$ vs. $73.9 \pm 12.0 \%)$ of Luminal A cancers were significantly lower than those of Luminal B cancers $(p<.05)$. The AUCs of T1, T2, and PD for the differentiation of Luminal B cancers from Luminal A cancers were 0.83 (95\% Cl: $0.61-0.95), 0.75$ (95\% Cl: $0.52-0.90)$, and 0.75 (95\% Cl: $0.53-0.91)$, respectively (Fig. 6). The T1 value had the highest AUC, followed by T2 and PD. The optimal sensitivity and specificity of the T1 value were $69 \%$ and $100 \%$.

\section{Discussion}

This study shows $\mathrm{T} 1, \mathrm{~T} 2$, and PD values, as derived from synthetic MRI, to be significantly and strongly correlated with $\mathrm{Ki}-67$. Ki-67 is considered to represent tumor proliferation status, with a higher Ki-67 being associated with an adverse clinical outcome [2]. High T2 values and high PD values may result in high signal intensity on T2-weighted images. That is, breast cancers with poor prognosis may show high signal intensity on T2-weighted images. This finding is in good agreement with prior studies. Uematsu et al. and others reported that tripe negative breast cancers (a subgroup of ER-negative breast cancers) had high signal intensity on T2-weighted images due to necrosis [10-13].

A correlation between high signal intensity on T2-weighted images and tumor grade in other subtypes of breast cancers has also been reported $[14,15,21]$. In those studies, it was postulated that edema with corresponding high signal intensity on $\mathrm{T} 2$-weighted images was possibly induced by angiogenesis as well as necrosis $[14,15,21,22]$. Seo et al. reported that $T 2 *$ relaxation time was significantly longer in higher histologic grades, the latter being correlated with high signal intensity on T2-weighted imaging [16].

However, most of these previous studies involved subjective qualitative analyses, which makes it difficult to apply their results in clinical practice for assessing hormone receptor status or subgroup categorization of ER-positive breast cancers. Recent advances in quantitative MRI enable the acquisition of both MR images and quantitative MR data within a single scan $[7,8,23]$. Synthetic MRI enables us to appreciate subtle quantitative MRI value differences, which cannot be seen by the radiologists' eyes alone. In addition, synthetic MRI can measure T1 values that cannot be assessed on T2-weighted images. In this study, the T2 value achieved an AUC of 0.87 for the differentiation of ER status. The T1 value reached an AUC of 0.83 for the discrimination of Luminal B type from Luminal A type breast cancers. 
Increased water content related to necrosis or edema may cause $\mathrm{T} 1$ elongation in Luminal type B breast cancers [24]. Matsuda et al. [23] reported that mean T1, mean T2, and mean PD in Luminal B type were higher than those in Luminal A type, however without any significant difference between the two groups. Because of fear of partial volume effect, only patients with lesions larger than $15 \mathrm{~mm}$ were analyzed in the present study, while the mean size of breast cancers in Matsuda et al.'s study was $17 \mathrm{~mm}$ in Luminal A type and $20 \mathrm{~mm}$ in Luminal A type [23]. We speculate that partial volume effect of surrounding tissue might have affected Matsuda et al.'s results and explain why a statistical significance was not achieved in their study.

The relationship between $\mathrm{Ki}-67$ and tumor grade with the ADC value has been reported by some previous studies $[11,14,23,25,26]$. In the present study, however, there was no significant correlation between ADC and Ki-67. This finding is similar to that reported by Kim et al [14]. Kim et al. reported that highly aggressive tumors may outgrow the oxygen supply of their vascular system, resulting in necrosis and decreased cellularity $[14,27]$. In high grade tumors, a higher tumor grade often goes hand in hand with a higher $A D C$ value $[11,14,26]$. In contrast, in relatively well-differentiated tumors, moderately proliferative cancers tend to have a higher cellularity than low proliferative tumors and generally have a lower ADC [25]. This paradoxical phenomenon can cause confusion in subtype prediction based on diffusionweighted imaging. Contrary to the ADC, both $T 1, T 2$, and PD values of high proliferative tumors were higher than those of low proliferative tumors. Because T1, T2, and PD value assessments do not experience this paradoxical phenomenon, we believe they are more useful than ADC measurements in this setting.

There are several limitations in this study. First, the number of patients in this study was relatively small. Second, 10 out of 28 patients underwent neoadjuvant chemotherapy and only their biopsy specimens could be used for analysis. A significant difference in $\mathrm{Ki}-67$ has been reported between specimens obtained at preoperative biopsy and surgery [28]. However, breast cancer patients with very high Ki-67 values very often undergo preoperative chemotherapy and surgical specimens without previous chemotherapy are hardly ever obtained [29]. In addition, in the 19 cancers for which both surgical and biopsy specimens were available, the correlation coefficients of quantitative MR values vs. Ki- 67 were similar between surgical and biopsy specimens. Third, because of the risk of partial volume effects on quantitative MRI measurements, only large mass lesions were included in this study. Our finding may not be applicable to non-mass lesions or smaller lesions such as ductal carcinoma in situ [23].

In conclusion, quantitative values from synthetic MRI significantly correlate with subtypes of invasive breast cancer and may classify subtypes with reasonably good accuracy.

\section{Declarations}

\section{Acknowledgements}

The authors would like to thank patients who took part in this study. This work was supported by Japan society for the promotion of science KAKENHI Grant Number 19 K08131. 


\section{Funding}

This work was supported by Japan society for the promotion of science KAKENHI Grant Number 19 K08131.

\section{Conflicts of interest}

The authors have no conflicts of interest to disclose.

\section{Ethical approval}

The institutional review board of Tokai University approved this study prior to recruiting participants.

\section{Consent to participate}

This study was performed in accordance with the Helsinki Declaration and written informed consent including publication was obtained from all participants prior to participation.

\section{Availability of data and material}

The datasets used and/or analyzed during the current study are available from the corresponding author on reasonable request.

\section{Code availability}

Not applicable' for that section.

\section{Author contributions}

All authors contributed to the study conception and design. Material preparation, data collection and analysis were performed by Toshiki Kazama, Noriko Nakamura and Nobue Kumaki. The first draft of the manuscript was written by Toshiki Kazama, Taro Takahara and Thomas C. Kwee. All authors commented on previous versions of the manuscript. All authors read and approved the final manuscript.

\section{References}

1. Siegel RL, Miller KD, Fuchs HE, Jemal A (2021) Cancer Statistics, 2021. CA Cancer J Clin 71:7-33. https://doi.org/10.3322/caac.21654

2. Coates AS, Winer EP, Goldhirsch A et al (2015) Tailoring therapies-improving the management of early breast cancer: St Gallen International Expert Consensus on the Primary Therapy of Early Breast Cancer 2015. Ann Oncol 26:1533-1546. https://doi.org/10.1093/annonc/mdv221

3. Goldhirsch A, Winer EP, Coates AS et al (2013) Personalizing the treatment of women with early breast cancer: highlights of the St Gallen International Expert Consensus on the Primary Therapy of Early Breast Cancer 2013. Ann Oncol 24:2206-2223. https://doi.org/10.1093/annonc/mdt303 
4. Niikura N, Masuda S, Kumaki N et al (2014) Prognostic significance of the Ki67 scoring categories in breast cancer subgroups. Clin Breast Cancer 14:323-329 e323.

https://doi.org/10.1016/j.clbc.2013.12.013

5. Niikura N, Liu J, Hayashi $\mathrm{N}$ et al (2012) Loss of human epidermal growth factor receptor 2 (HER2) expression in metastatic sites of HER2-overexpressing primary breast tumors. J Clin Oncol 30:593599. https://doi.org/10.1200/JC0.2010.33.8889

6. Niikura N, Tomotaki A, Miyata $\mathrm{H}$ et al (2016) Changes in tumor expression of HER2 and hormone receptors status after neoadjuvant chemotherapy in 21,755 patients from the Japanese breast cancer registry. Ann Oncol 27:480-487. https://doi.org/10.1093/annonc/mdv611

7. Arita Y, Takahara T, Yoshida S et al (2019) Quantitative Assessment of Bone Metastasis in Prostate Cancer Using Synthetic Magnetic Resonance Imaging. Invest Radiol 54:638-644. https://doi.org/10.1097/RLI.0000000000000579

8. Warntjes JB, Leinhard OD, West J, Lundberg P (2008) Rapid magnetic resonance quantification on the brain: Optimization for clinical usage. Magn Reson Med 60:320-329. https://doi.org/10.1002/mrm.21635

9. Jung Y, Gho SM, Back SN, Ha T, Kang DK, Kim TH (2018) The feasibility of synthetic MRI in breast cancer patients: comparison of T2 relaxation time with multiecho spin echo T2 mapping method. $\mathrm{Br}$ J Radiol:20180479. https://doi.org/10.1259/bjr.20180479

10. Uematsu T, Kasami M, Yuen S (2009) Triple-negative breast cancer: correlation between MR imaging and pathologic findings. Radiology 250:638-647. https://doi.org/10.1148/radiol.2503081054

11. Youk JH, Son EJ, Chung J, Kim JA, Kim EK (2012) Triple-negative invasive breast cancer on dynamic contrast-enhanced and diffusion-weighted MR imaging: comparison with other breast cancer subtypes. Eur Radiol 22:1724-1734. https://doi.org/10.1007/s00330-012-2425-2

12. Sung JS, Jochelson MS, Brennan S et al (2013) MR imaging features of triple-negative breast cancers. Breast J 19:643-649. https://doi.org/10.1111/tbj.12182

13. Dogan BE, Gonzalez-Angulo AM, Gilcrease M, Dryden MJ, Yang WT (2010) Multimodality imaging of triple receptor-negative tumors with mammography, ultrasound, and MRI. AJR Am J Roentgenol 194:1160-1166. https://doi.org/10.2214/AJR.09.2355

14. Kim SY, Kim EK, Moon HJ et al (2018) Association among T2 signal intensity, necrosis, ADC and Ki67 in estrogen receptor-positive and HER2-negative invasive ductal carcinoma. Magn Reson Imaging 54:176-182. https://doi.org/10.1016/j.mri.2018.08.017

15. Yuen S, Uematsu T, Kasami M et al (2007) Breast carcinomas with strong high-signal intensity on T2weighted MR images: pathological characteristics and differential diagnosis. J Magn Reson Imaging 25:502-510. https://doi.org/10.1002/jmri.20845

16. Seo M, Ryu JK, Jahng GH et al (2017) Estimation of T2* Relaxation Time of Breast Cancer: Correlation with Clinical, Imaging and Pathological Features. Korean J Radiol 18:238-248. https://doi.org/10.3348/kjr.2017.18.1.238 
17. WHO Classification of Tumours Editorial Board (2019) World Health Organization classification of tumours. Breast Tumours. International Agency for Research on Cancer, Lyon

18. Hammond ME, Hayes DF, Dowsett M et al (2010) American Society of Clinical Oncology/College of American Pathologists guideline recommendations for immunohistochemical testing of estrogen and progesterone receptors in breast cancer. Arch Pathol Lab Med 134:907-922. https://doi.org/10.1043/1543-2165-134.6.907

19. Curigliano G, Burstein HJ, Winer EP et al (2017) De-escalating and escalating treatments for earlystage breast cancer: the St. Gallen International Expert Consensus Conference on the Primary Therapy of Early Breast Cancer 2017. Ann Oncol 28:1700-1712.

https://doi.org/10.1093/annonc/mdx308

20. Dowsett M, Nielsen TO, A'Hern R et al (2011) Assessment of Ki67 in breast cancer: recommendations from the International Ki67 in Breast Cancer working group. J Natl Cancer Inst 103:1656-1664. https://doi.org/10.1093/jnci/djr393

21. Renz DM, Baltzer PA, Bottcher J et al (2008) Magnetic resonance imaging of inflammatory breast carcinoma and acute mastitis. A comparative study. Eur Radiol 18:2370-2380. https://doi.org/10.1007/s00330-008-1029-3

22. Baltzer PA, Yang F, Dietzel M et al (2010) Sensitivity and specificity of unilateral edema on T2W-TSE sequences in MR-Mammography considering 974 histologically verified lesions. Breast J 16:233239. https://doi.org/10.1111/j.1524-4741.2010.00915.x

23. Matsuda M, Kido T, Tsuda T et al (2020) Utility of synthetic MRI in predicting the Ki-67 status of oestrogen receptor-positive breast cancer: a feasibility study. Clin Radiol 75:398 e391-398 e398. https://doi.org/10.1016/j.crad.2019.12.021

24. Crooks LE, Hylton NM, OrtendahI DA, Posin JP, Kaufman L (1987) The value of relaxation times and density measurements in clinical MRI. Invest Radiol 22:158-169. https://doi.org/10.1097/00004424198702000-00014

25. Mori N, Ota H, Mugikura S et al (2015) Luminal-type breast cancer: correlation of apparent diffusion coefficients with the Ki-67 labeling index. Radiology 274:66-73. https://doi.org/10.1148/radiol.14140283

26. Martincich L, Deantoni V, Bertotto I et al (2012) Correlations between diffusion-weighted imaging and breast cancer biomarkers. Eur Radiol 22:1519-1528. https://doi.org/10.1007/s00330-012-2403-8

27. Leek RD, Landers RJ, Harris AL, Lewis CE (1999) Necrosis correlates with high vascular density and focal macrophage infiltration in invasive carcinoma of the breast. Br J Cancer 79:991-995. https://doi.org/10.1038/sj.bjc.6690158

28. Romero Q, Bendahl PO, Klintman M et al (2011) Ki67 proliferation in core biopsies versus surgical samples - a model for neo-adjuvant breast cancer studies. BMC Cancer 11:341. https:// doi.org/10.1186/1471-2407-11-341

29. Mauri D, Pavlidis N, loannidis JP (2005) Neoadjuvant versus adjuvant systemic treatment in breast cancer: a meta-analysis. J Natl Cancer Inst 97:188-194. https://doi.org/10.1093/jnci/dji021 


\section{Tables}

Table 1. Characteristics of cancers according to ER status

Age (y)

Diameter (mm)

Ki-67

$\mathrm{T} 1$ (msec)

$\mathrm{T} 2$ (msec)

PD (\%)

$69.3 \pm 12.7$

$72.7 \pm 15.7$

Data are presented as mean \pm standard deviation
$92.1 \pm 13.0$

$74.5 \pm 5.8$

$60.7 \pm 8.0$

$27.7 \pm 9.8$

.79

$51.7 \pm 23.2$

.01

$1331 \pm 201$

.08

.006

39

$E R$, estrogen receptor; $P D$, proton density

Table 2. Correlation of quantitative MR values with $\mathrm{Ki}-67$ 
Pearson's coefficient PValue

All $(\mathrm{n}=29)$

$\begin{array}{lcc}\text { T1 } & 0.75 & <.0001 \\ \text { T2 } & 0.69 & <.0001 \\ \text { PD } & 0.60 & .0006 \\ \text { ADC } & 0.11 & .57\end{array}$

Resected cancers $(n=19)$

Biopsy Ki-67 $(n=19)$

T1

T2

PD

Resected specimen Ki-67 $(n=19)$

T1

$\mathrm{T} 2$

PD
0.72

0.74

0.57

0.74

0.72

0.59
.0005

.0003

.01

.0005

.008

$\mathrm{ADC}$, apparent diffusion coefficient; $\mathrm{ER}$, estrogen receptor, $\mathrm{PD}$, proton density

Table 3. Characteristics of Luminal A and Luminal B cancers 


$$
\text { Luminal A group }(\mathrm{n}=10)
$$

Age (y)

Diameter (mm)

Ki-67

$\mathrm{T} 1$ (msec)

$\mathrm{T} 2$ (msec)

PD (\%)

$63.4 \pm 11.4$

$8.0 \pm 2.6$

$991 \pm 132$

$65.2 \pm 12.6$
Luminal B group $(n=13)$

$57.9 \pm 12.5$

$28.8 \pm 16.9$

$78.5 \pm 15.8$

$73.9 \pm 12.0$
.90

P Value

.005

.001

.008

.047

.041

Data are presented as mean \pm standard deviation

$\mathrm{PD}$, proton density

Figures

\section{T2 and ER status}

ER (-)

$\left.\begin{array}{r}140 \\ 130- \\ 120 \\ 110- \\ 100- \\ 90 \\ 80- \\ 70- \\ 60- \\ 50 \\ 40\end{array}\right]$

ER (+) 


\section{Figure 1}

Boxplots displaying the distribution of T2 values according to ER status. The box extends from the 25th to the 75th percentile. The line is the median value. T2 of ER-negative cancers is significantly higher than that of ER-positive cancers $(p<.01)$.

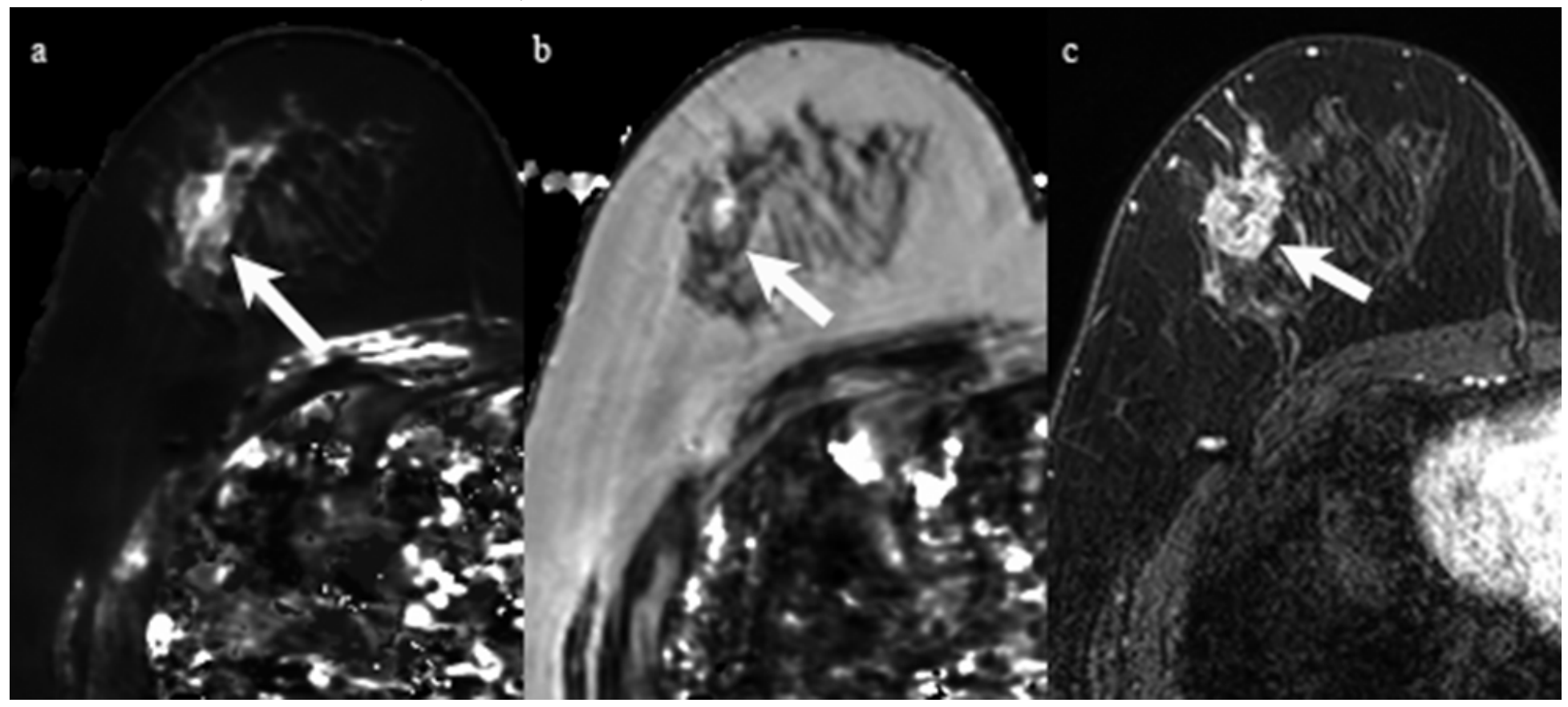

Figure 2

A 59-year-old woman with IDC in the right breast. Immunohistochemical staining showed negativity for estrogen receptor and Ki-67 of $60 \%$. a: T1 map shows a high signal mass. Mean T1 of the mass is 1477 msec. b: T2 map shows a high signal mass. Mean T2 of the mass is $96 \mathrm{msec}$. c: Corresponding contrast enhanced MR image shows a mass with heterogeneuous enhancement.

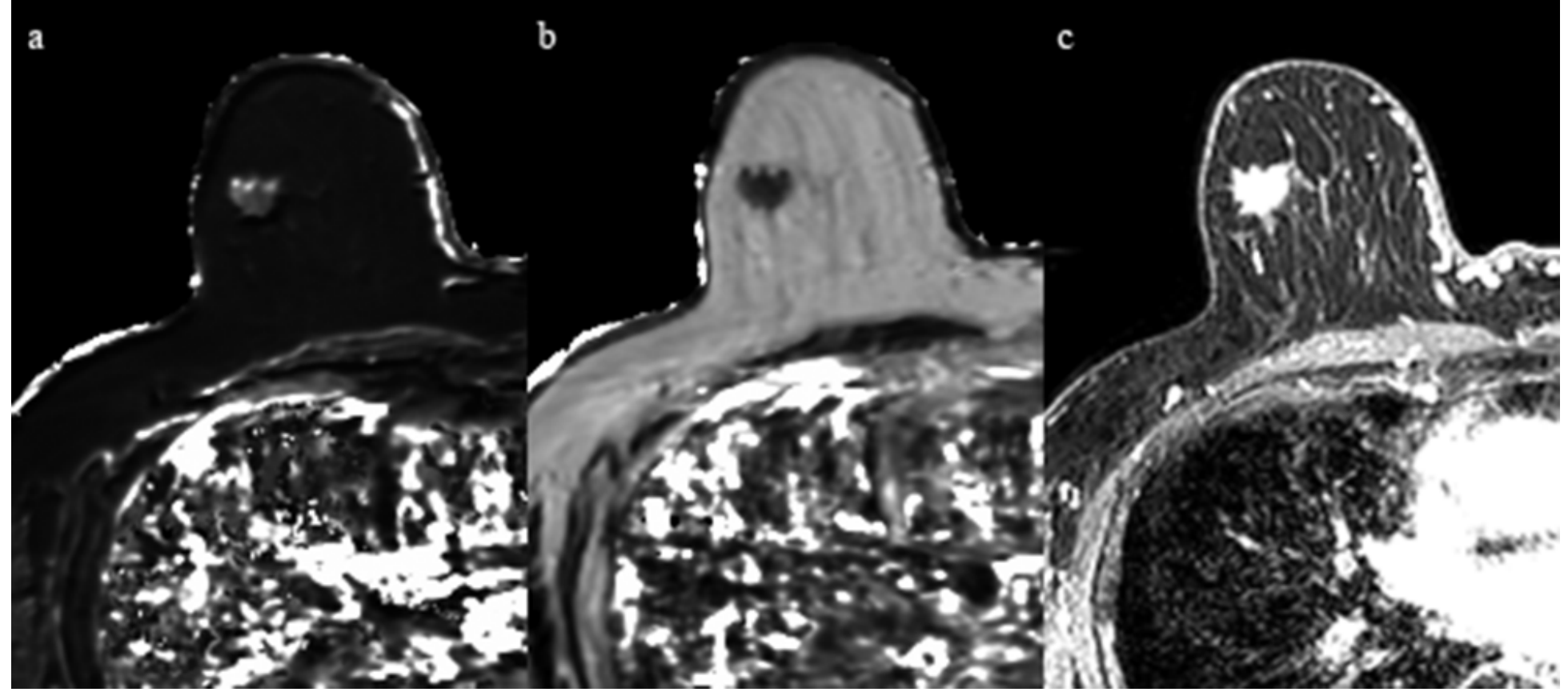


Figure 3

A 69-year-old woman with IDC in the right breast. Immunohistochemical staining showed positivity for estrogen receptor and Ki-67 of 10\%. a: T1 map shows an intermediate signal mass. Mean T1 of the mass is $986 \mathrm{msec}$. b: T2 map shows a low signal mass. Mean T2 of the mass is $61 \mathrm{msec}$. c: Corresponding contrast enhanced MR image shows a mass with homogeneous enhancement.

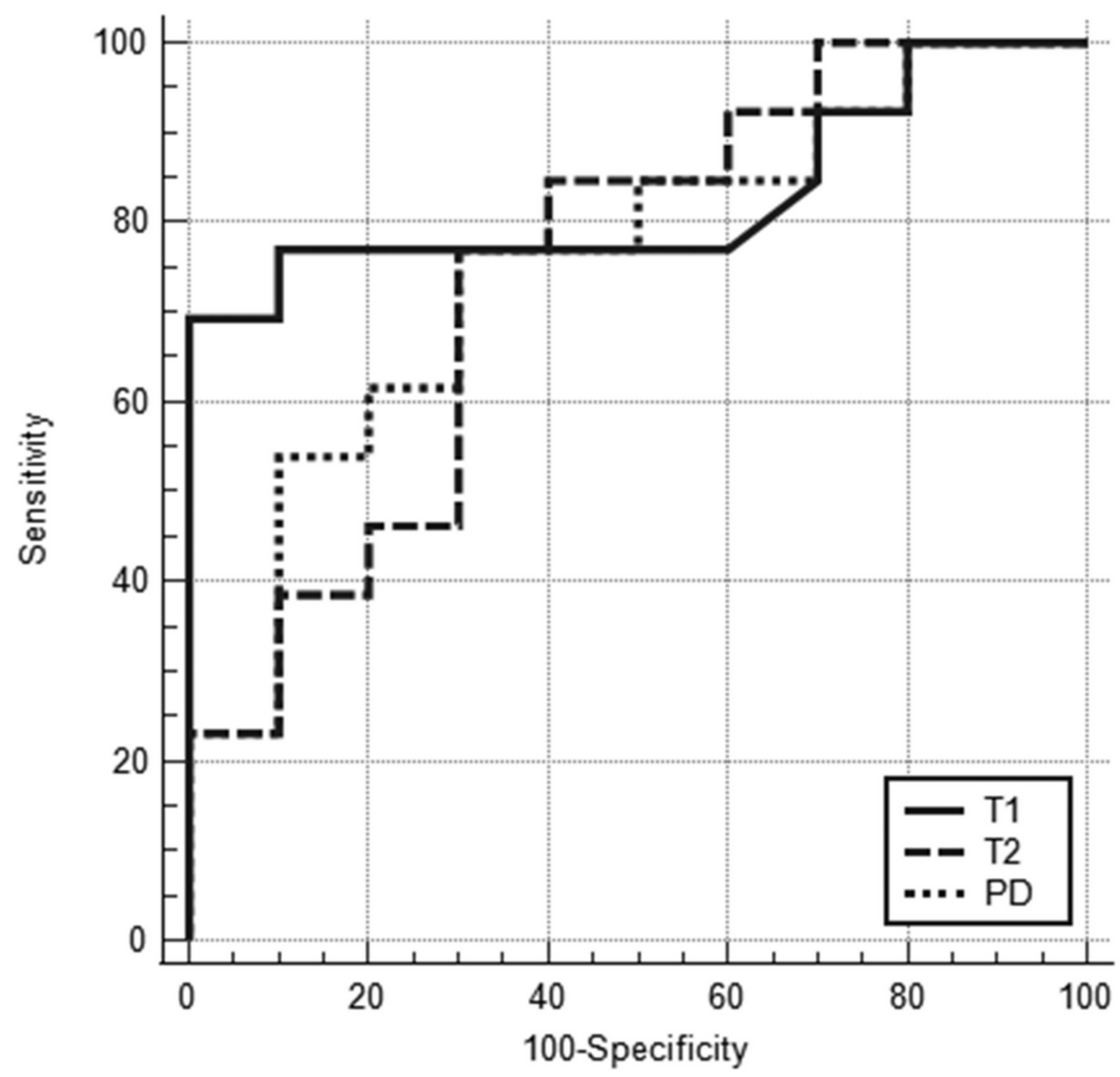

Figure 4

ROC curve of T1, T2, and PD values for differentiating ER-negative cancers from ER-positive cancers shows that the areas under the ROC curve are 0.74 (95\% confidence interval (Cl): 0.54-0.88), 0.87 (95\% Cl: 0.69-0.97), and 0.62 (95\% Cl: 0.42-0.79), respectively. 


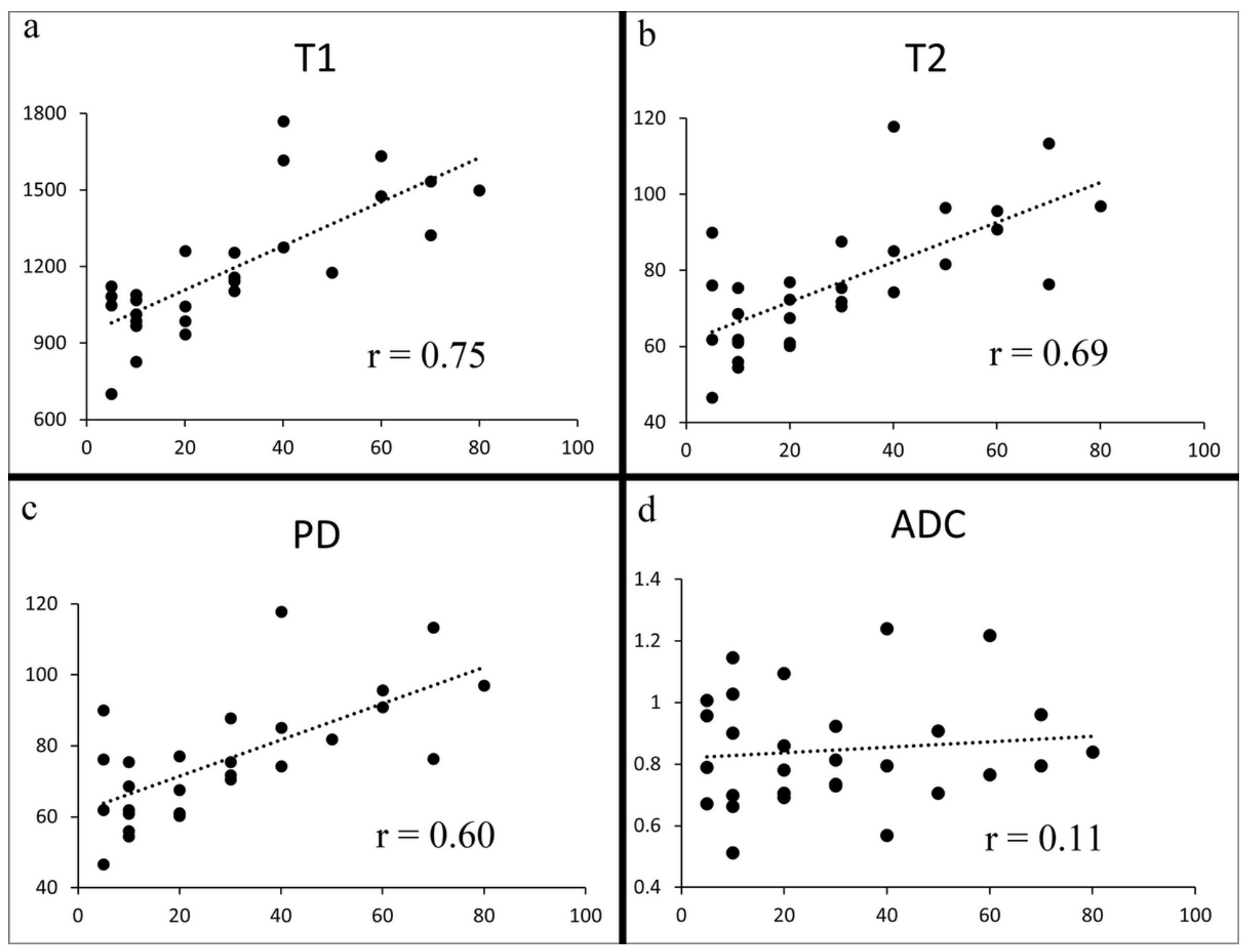

Figure 5

Scatter plots of Ki-67 vs. T1 (a), T2 (b), PD (c), and ADC (d). Significant correlations are observed between $\mathrm{Ki}-67$ and T1, between Ki-67 and T2, and between Ki-67 and PD (a-c) $(p<.001)$. No significant correlation is seen between $\mathrm{Ki}-67$ and ADC ( $p=.57)(d)$. 


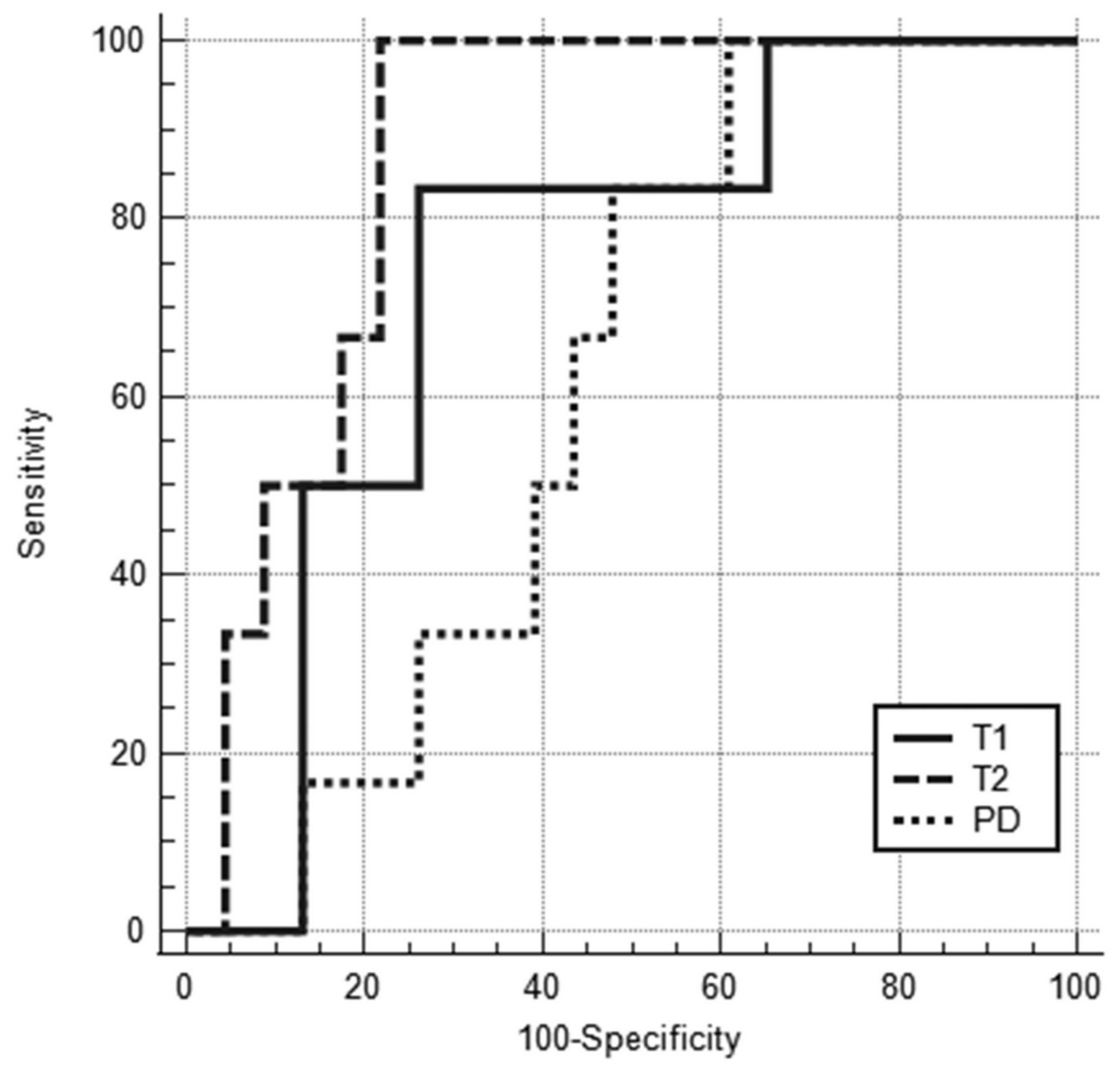

Figure 6

ROC curves for T1, T2, and PD values for differentiating Luminal A from the Luminal $B$ cancers shows that the areas under the ROC curve are 0.83 (95\% Cl: $0.61-0.95), 0.75$ (95\% Cl: $0.52-0.90)$, and 0.75 (95\% Cl: 0.53-0.91), respectively. 